\title{
Processing speed, laterality patterns, and memory encoding as a function of hemispheric dominance
}

\author{
SHARON COLEMAN and ROBERT ZENHAUSERN \\ St. John's University, Jamaica, New York 11439
}

\begin{abstract}
Hemispheric dominance is a cognitive style based on the differential ability to use the capabilities of the right or the left hemisphere. Subjects, classified as either right or left dominant, performed a discriminative reaction time task that involved a memory component. The two groups were shown to differ on processing speed and encoding strategies.
\end{abstract}

There is growing evidence of the existence of a cognitive style, encompassing many areas of human performance, that depends on the differential use of the capabilities of the two cerebral hemispheres. While both hemispheres can be involved in the performance of all tasks (with the possible exception of language production, which is typically the province of the left hemisphere, at least in right-handed people), the left hemisphere seems to have more verbal and sequential abilities, while the right hemisphere excels in visualspatial tasks. Individuals who are more efficient at using the parallel holistic processing style of the right hemisphere have been termed right-hemispheric dominant; those who are more efficient at using the sequential verbal abilities of the left hemisphere have been called left-hemispheric dominant.

One of the earliest means of identifying these two thinking styles was through the use of eye movements in response to questions (e.g., Day, 1964; Duke, 1968; Teitelbaum, 1954). There was considerable controversy and lack of consistency, however, from experiment to experiment until Gur and Gur (1977), and Gur, Gur, and Harris (1975) clarified the issue by showing that the position of the questioner was a crucial variable. When the experimenter was not in face-to-face contact with the subject, the eye movements reflected laterality; that is, the eyes moved contralaterally to the hemisphere most efficient at processing that kind of information. In the face-to-face situation, however, the eye movements reflected dominance; that is, the eyes moved consistently in one direction, independent of the type of question. Sackeim, Packer, and Gur (1977) have shown that susceptibility to subliminal perception depends on the adoption of an organizational set that is consistent with the cognitive style of the subjects, as measured by eye movements.

This paper is based on the PhD dissertation of the first author submitted to St. John's University. Requests for reprints should be sent to the second author at SB-15 Marillac, St. John's University.
Hellige (1975) differentiated subjects on the basis of the form of their classically conditioned eye blinks. The V-type individual has characteristics similar to those postulated for the left hemisphere, while the C-type individuals have characteristics more typically associated with the right hemisphere. Using a simultaneous same-different reaction time task for letters, the two conditioning types produced different laterality patterns (performance in the left as opposed to the right hemisphere), with the $\mathrm{V}$ type showing a greater differential performance between the two hemispheres. It should be noted, however, that the V-type subjects showed superior right-hemisphere performance for verbal material.

Arndt and Berger (Note 1), using tests designed to measure the spatial-holistic abilities of the right hemisphere and the verbal-analytic abilities of the left hemisphere, tested the two resulting groups on a lateralized discrimination task. Stimuli were either letters or faces, and the dependent variable was response latency. Although there was no interaction of Cognitive Style by Hemisphere, the spatial-holistic group had consistently faster reaction times than the verbal-analytic group. This was in contrast with Hellige (1975), who found differential laterality patterns, but no differences in group performance.

Cognitive tempo is the term used to describe performance on Kagan's (1965) matching familiar figure test. Impulsives are individuals who give fast but inaccurate responses; reflectives are accurate, but slow. The subject's task is to determine which of the comparison stimuli is identical to a standard, with the differences among the comparison stimuli based on comparatively minor differences in details among the figures. Zelniker and Jeffrey (1977) developed a new set of stimuli in which the differences among the comparison stimuli depended on overall global differences rather than on differences in detail. When this new test was administered to a previously determined group of impulsives and reflectives, the errors of the impulsives decreased and the errors of the reflectives increased, leading to a 
slight but nonsignificant difference in favor of the impulsives. These results are consistent with the hypothesis that impulsives process in the holistic style of rightdominant individuals and reflectives process in the sequential style of left-dominant individuals.

Torrance, Reynolds, Riegel, and Ball (1977) have developed a paper-and-pencil subjective report scale ("Your Style of Learning and Thinking") to measure a subject's preference for right-hemisphere as opposed to left-hemisphere functions. In terms of reliability, construct validity, and concurrent validity, the test shows considerable potential as a measure of hemispheric dominance.

Fadely and Hosler (1979) have extended the concept of hemispheric dominance to school and preschool children. Their major emphasis is that extremely rightdominant children are at a disadvantage in school and they may be incorrectly diagnosed as brain damaged or hyperactive.

Zenhausern (1978) has proposed a model of hemispheric dominance based on the hypothesis that for a right-dominant individual, visual images are essential to thinking and deductive reasoning is the preferred mode; for left-dominant individuals, visual images play an ancillary role and inductive reasoning is preferred. Several studies have provided support for the model. Zenhausern and Repetti (Note 2) found that subjects who reported they thought in pictures scored higher on the right scale of the Torrance et al. (1977) test than subjects who reported they did not think in pictures. The reverse pattern was found on the test for those subjects who reported they did not think in pictures. Zenhausern and Gebhardt (1979) found differential memory performance for right- and left-hemispheric dominant subjects. While they found rather complex interaction effects, there was a consistent finding of greater retention of material under auditory rather than visual input for left-dominant individuals and the reverse effect for right-dominant individuals. Zenhausern and Nickel (in press) have found that right-dominant individuals outperformed left-dominant individuals in maze learning in terms of both time and errors.

The purpose of this experiment was to examine the difference between left- and right-dominant individuals on a lateralized reaction time task. The groups were compared on both overall performance and laterality patterns.

\section{METHOD}

\section{Subjects}

Sixteen male and 16 female graduate and undergraduate students served as subjects in this experiment. On the basis of agreement between "Your Style of Learning and Thinking" (Torrance et al., 1977) and a questionnaire developed by Zenhausern and Repetti (Note 2), these subjects were classified as either right- or left-hemispheric dominant. Thus, four equalsized groups were formed: male and female, left- and righthemispheric dominant.

\section{Apparatus and Materials}

Stimuli. There were two kinds of stimuli, targets and probes, half of each consisting of words and half of pictures. Thus 24 words were selected from the list provided by Paivio, Yuille, and Madigan (1968) and consisted of words rated high in concreteness (mean $=5.8)$ and imagery value $($ mean $=6.2)$ on a 7-point scale. The 24 pictures consisted of simple line drawings of the same objects represented by the words. The target stimuli consisted of either two words or two pictures, centrally located above and below the point of fixation. The probe stimuli consisted of a single word or picture located to the right or left of fixation. All stimuli took the form of Kodak high-speed Ektachrome slides. When projected to the subject, the picture stimuli subtended a visual angle of $4 \mathrm{deg}$ in length and $2 \mathrm{deg}$ in height, while the corresponding angles for the word stimuli were $4 \mathrm{deg}$ and $2 \mathrm{deg} 4 \mathrm{~min}$, respectively. The visual angle between the two targets was $1 \mathrm{deg} 10 \mathrm{~min}$, and the probes were presented $1 \mathrm{deg} 20 \mathrm{~min}$ to the left or right of fixation.

The 64 stimuli were arranged in four blocks, each consisting of 2 vertically positioned target stimuli alternating with a probe, for a total of 32 pairs. The four blocks were homogeneous with respect to the target and probe form: (1) picture-picture, picture targets and picture probes; (2) picture-word, picture targets and word probes; (3) word-picture, word targets and picture probes; and (4) word-word, word targets and word probes. Within each block, an equal number of probes were presented in the left and in the right visual fields; half were of the same form or concept as one of the two targets, and half were completely different. In addition, a practice block of 32 targets and 32 probes, consisting of 8 of each of the target and probe types, was developed.

Apparatus. The slides were rear-projected by means of a Kodak Model 750 Carousel projector, with exposure duration controlled by means of a Gerbrands electronic shutter. The subjects' response keys consisted of two double-throw, centeroff toggle switches, wired so that a forward throw indicated a "same" response and a backward throw indicated a "different" response. To control for any interaction of hemisphere of input with hemisphere of output, both switches were thrown by the two hands to indicate a decision. A BRS electronic logic program was used to control slide presentation, exposure duration, and measurement of reaction time in milliseconds by means of a Hunter Klockcounter. Accuracy of the same-different responses was verified by indicator lights on the logic modules.

\section{Procedure}

The subjects were exposed to the 32 practice trials to familiarize them with the task requirements. The four experimental blocks were presented in a randomized order across the 32 subjects. In all cases the following sequence was observed. The two centrally presented targets were exposed for $1 \mathrm{sec}$, followed by a 1-sec blank interval, and then a 125 -m sec exposure of a probe to one of the two visual fields. The millisecond timer started simultaneously with the onset of the probe and terminated with the response of the subject. Even though the probes were presented randomly to the left or right visual field, so as to control anticipatory eye movements, the subject was periodically warned to maintain a central fixation: a black dot on the rearprojection screen. The subject was positioned $24 \mathrm{in}$. from the screen, and a Bausch and Lomb chin rest was used to help control fixation and head movements. After completion of all four blocks, a blank slide was inserted in the projector, the intensity of the projector lamp was dimmed, and 32 simple reaction time trials to the onset of the light were obtained.

\section{RESULTS AND DISCUSSION}

The mean reaction time data were subjected to a reciprocal transformation and analyzed by means of a split-plot analysis of variance with hemispheric dominance and sex as between-subjects factors and target type, probe type, and hemisphere of presentation as within-subjects factors. Preliminary analysis indicated 
that, while "same" responses were significantly faster than "different" responses, there were no meaningful interactions involving this factor, and so it was collapsed in the main analysis.

\section{Hemispheric Dominance and Processing Speed}

The analysis of variance resulted in a significant $[F(1,28)=6.22, p<.05]$ effect of hemispheric dominance, with right dominants (mean $=752 \mathrm{msec}$ ) faster than left dominants (mean $=869 \mathrm{msec}$ ). Neither sex nor its interaction with dominance was significant: This faster reaction time was consistent with the effect reported by Arndt and Berger (Note 1) and, more indirectly, with the results of Zelniker and Jeffrey (1977) on cognitive tempo.

In order to explore the nature of the superiority of the right dominants, the simple reaction time means were used as a covariate in the main analysis. Preliminary analysis indicated significantly faster reaction times for right dominants $($ mean $=230)$ than for left dominants (mean $=287$ ), but no sex differences. The significant superiority of the right dominants on the discrimination task remained after the analysis of covariance. It is tempting to conclude that, in this type of task, with the motor component held constant, cerebral processing of right dominants is faster than the style of left dominants. As Boring (1957) pointed out, however, the problem with Donders' subtractive technique was that the nature of the task changes from simple to complex reaction time, and a simple additive model is not appropriate. Because of this possible change, it is unclear exactly what has been partialed out by the analysis of covariance.

It is clear, however, that for the task as a whole, the right dominants are faster than the left dominants. According to the Zenhausern (1978) model, as supported by Zenhausern and Repetti (Note 2), the difference between the two styles lies in whether thought is internally represented by pictures or words. A holistic parallel processing style is generally associated with the right hemisphere, and a pictoral representation is consistent with that style. The left hemisphere, on the other hand, is more sequential and logical in style, and a verbal representation is consistent with that style. These facts are consistent with the conclusion that the parallel processing style of the right-dominant individual is more efficient for this task than is the serial processing style of the left-dominant individual.

\section{Hemispheric Dominance and Laterality}

Reaction time was significantly faster $[F(1,28)=$ $32.94, p<.001]$ for probes presented to the left hemisphere (mean $=795)$ than for probes presented to the right hemisphere $($ mean $=827)$. Hemisphere of input did not interact significantly with target or probe type, indicating faster left-hemisphere processing for both words and pictures. Several studies (e.g., Hellige \& Cox, 1976; Kinsbourne, 1975) have shown that a concurrent verbal memory load influences laterality patterns, such that a left-hemisphere superiority is found for both verbal and nonverbal material. This result has been attributed to the selective activation of the left hemisphere by the memory load. In the present study, the target stimuli themselves served as the memory load, resulting in the left-hemisphere superiority. More recently, Hellige, Cox, and Litvak (1979) have shown that there is no alteration of laterality patterns with a concurrent nonverbal memory load. Since in this study even the pictoral target stimuli resulted in faster responses in the left hemisphere, it can be concluded that there was at least some verbal coding of this material.

While hemisphere of input did not interact with stimulus or probe type, there was a significant Dominance by Hemisphere of Input interaction $[F(1,28)=$ $5.04, p<.05]$. The means for this effect can be seen in Table 1. Simple effects indicated that performance in the left hemisphere was superior for both right- and leftdominant individuals, and right dominants had shorter latencies than left dominants in both hemispheres. The interaction arose because the superiority of the left hemisphere for left dominants $(56 \mathrm{msec})$ was four times larger than the difference for right dominants $(14 \mathrm{msec})$. These results seem to indicate that the activation of the left hemisphere due to the verbal memory load was stronger in left-dominant subjects than in right-dominant subjects. This conclusion is consistent with the proposed differences in processing styles between the two groups.

\section{Hemispheric Dominance and Dual Coding}

There was a significant interaction $[\mathrm{F}(1,28)=47.87$, $\mathrm{p}<.001]$ between target type and probe type. The means of this effect for right- and left-dominant individuals appear in Table 2. An analysis of the simple effects indicated that when words were probes, there were no differences between target types, but with pictures as probes, reaction time was significantly faster for picture targets than for word targets. Conversely, there were no differences between probe

Table 1

Mean Reaction Time (in Milliseconds) as a Function of Hemispheric Dominance and Hemisphere of Input

\begin{tabular}{ccc} 
& \multicolumn{2}{c}{ Hemisphere of Input } \\
\cline { 2 - 3 } Dominance & Right & Left \\
\hline Right & 757 & 743 \\
Left & 898 & 842 \\
\hline
\end{tabular}

Table 2

Mean Reaction Time (in Milliseconds) for Target and Probe Types as a Function of Hemispheric Dominance

\begin{tabular}{cccccc}
\hline & \multicolumn{2}{c}{ Picture Target } & & \multicolumn{2}{c}{ Word Target } \\
\cline { 2 - 3 } \cline { 5 - 6 } Dominance & $\begin{array}{c}\text { Picture } \\
\text { Probe }\end{array}$ & $\begin{array}{c}\text { Word } \\
\text { Probe }\end{array}$ & & $\begin{array}{c}\text { Picture } \\
\text { Probe }\end{array}$ & $\begin{array}{c}\text { Word } \\
\text { Probe }\end{array}$ \\
\hline Right & 682.0 & 777.0 & & 789.0 & 753.0 \\
Left & 829.0 & 888.0 & & 888.0 & 874.0 \\
Mean & 755.5 & 832.5 & & 838.5 & 813.5 \\
\hline
\end{tabular}


types for word targets, but reaction time to picture probes was significantly faster than word probes for picture targets. Paivio (1976) has proposed a dual coding model of memory encoding, in which it is postulated that material is encoded and can be retrieved in both verbal and pictoral form. If it is assumed that pictures are dual coded, then word probes should be equally effective for both picture and word targets. If, on the other hand, words were not dual coded, a picture probe would be more effective for a picture target than a word target. More likely, words are dual coded but the pictorial form is idiosyncratic to a subject and not identical to the picture stimuli used in this study. That is, it is highly unlikely that an individual's internal representation of a dog would be identical to that of the artist who drew the stimulus picture. Thus, either words are not dual coded or the dual coding is not effective for this task.

Target type and probe type interacted with hemispheric dominance to produce a significant three-way interaction $[F(1,28)=5.62, p<.05]$. An analysis of the simple, simple effects indicated that the same Target by Probe interaction was found for both rightand left-dominant subjects, but the effect was stronger for the right dominants. The most interesting aspect of this interaction, however, was the lack of a significant difference of hemispheric dominance in the word target/picture probe condition, as opposed to the other three target-probe conditions, in which the right dominants were significantly faster than the left dominants. This condition resulted in the slowest reaction time for the right dominants, but not for the left dominants. The faster processing style of the right dominants may depend on the use of pictoral encoding and retrieval. In the condition in which a word stimulus is followed by a picture probe, there is more than enough time to code the word in picture form. When the picture probe is presented, however, it is not identical to the idiosyncratic internal representation of the subject, and the necessary conversion may be responsible for the increased latency. The left dominants, on the other hand, may use a verbal encoding and retrieval style that would be handicapped less by this condition.

\section{SUMMARY}

The results of this study indicate that right- and leftdominant individuals use different processing styles as measured by speed, laterality, and encoding. It is also clear that these differences are highly task dependent, as can be seen from a comparison with the results of Hellige (1975) and Arndt and Berger (Note 1). Differences exist, but the nature of these differences is dependent on the specific requirements of the experiment. What seems to be emerging is the existence of two distinct groups: one which is more efficient in tasks that demand a parallel processing mode, and another which is more efficient at tasks that demand a sequential processing mode.

\section{REFERENCE NOTES}

1. Arndt, L., \& Berger, D. Cognitive mode and asymmetry in cerebral functioning. Paper presented at the 85th convention of the American Psychological Association, San Francisco, 1977.

2. Zenhausern, R., \& Repetti, J. A comparison of two measures of cerebral dominance. Paper presented at the Eastern Psychological Association, Philadelphia, 1979.

\section{REFERENCES}

Boring, E. A history of experimental psychology. New York: Appleton-Century-Crofts, 1957.

DAY, M. An eye movement phenomenon relating to attention, thought, and anxiety. Perceptual and Motor Skills, 1964, 19, 443-446.

Duke, J. Lateral eye-movement behavior. Journal of General Psychology, 1968, 78, 189-195.

FAdely, J., \& Hosler, V. Understanding the alpha child at home and school. Springfield, Ill: Thomas, 1979.

Gur, R. E., \& Gur, R. C. Correlates of conjugate lateral eye movements in man. In S. Harnad, R. Doty, L. Goldstein, J. Jaynes, \& S. Krauthamer (Eds.), Lateralization in the nervous system. New York: Academic Press, 1977.

Gur, R. E., Gur, R. C., \& Harris, L. Cerebral activation, as measured by subjects' lateral eye movements, is influenced by experimenter location. Neuropsychologia, 1975, 13, 35-44.

Hellige, J. Hemispheric processing differences revealed by differential conditioning and reaction time performance. Journal of Experimental Psychology: General, 1975, 104, 309-326.

Hellige, J., \& Cox, P. Effects of concurrent verbal memory on recognition of stimuli from the left and right visual fields. Journal of Experimental Psychology: Human Perception and Performance, 1976, 2, 210-221.

Hellige, J., Cox, P., \& Litvak, L. Information processing in the cerebral hemispheres: Selective hemispheric activation and capacity limitations. Journal of Experimental Psychology: General, 1979, 108, 251-279.

KAGAN, J. Impulsive and reflective children: Significance of conceptual tempo. In J. Krumboltz (Ed.), Learning and the educational process. Chicago: Rand McNally, 1965.

Kinsbourne, M. The mechanism of hemispheric control of the lateral gradient of attention. In P. Rabbit \& S. Dornic (Eds.), Attention and performance $V$. New York: Academic Press, 1975.

PAIvio, A. Imagery in recall and recognition. In J. Brown (Ed.), Recall and recognition. New York: Wiley, 1976.

Paivio, A., Yuille, J., \& Madigan, S. Concreteness, imagery, and meaningfulness values for 925 nouns. Journal of Experimental Psychology Monograph, 1968, 76(1, Pt. 2).

Sackeim, H., Packer, I., \& GuR, R. C. Hemisphericity, cognitive set, and susceptibility to subliminal perception. Journal of Abnormal Psychology, 1977, 86, 624-630.

Teitelbaum, H. Spontaneous rhythmic ocular movements: Their possible relationship to mental activity. Neurology, 1954, 4, 350-354.

Torrance, E., Reynolds, C., Riegel, T., \& Ball, O. Your style of learning and thinking, Forms A and B. Gifted Child Quarterly, 1971, 21, 563-573.

ZELNIKER, T., \& JEFFREY, W. Reflective and impulsive children: Strategies of information processing underlying differences in problem-solving. Monographs of the Society for Research and Child Development, 1977, 168, 5.

ZENHAUSERN, R. Imagery, cerebral dominance, and style of thinking: A unified field model. Bulletin of the Psychonomic Society, 1978, 12, 381-384.

Zenhausern, R., \& Gebhardt, M. Hemispheric dominance in recall and recognition. Bulletin of the Psychonomic Society, 1979, 14, 71-73.

Zenhausern, R., \& Nickel, L. Hemispheric dominance and maze learning. Bulletin of the Psychonomic Society, in press.

(Received for publication August 14, 1979.) 